\title{
PLANEAMIENTO AMBIENTAL A ESCALA DE DETALLE: MICRORRESERVAS DE FLORA EN LA COMUNIDAD VALENCIANA
}

\author{
Ascensión Padilla \\ Alfredo Ramón
}

\section{RESUMEN}

La planificación del medio físico tiene, entre sus objetivos prioritarios, la aplicación de una política preventiva, capaz de evitar el deterioro del medio ambiente. La preservación de espacios de calidad ambiental lleva consigo la creación de figuras de protección dotadas de un cuerpo legal que asegura su gestión funcional. La actitud política, al respecto, ha variado a lo largo de la historia, impuesta por criterios económicos e influida por aspectos culturales y por la propia evolución científica en el conocimiento del medio ambiente. La aparición de las microrreservas de flora en la Comunidad Valenciana responde a una nueva estrategia en materia de protección, basada en criterios científicos y ecológicos, siendo, además, una medida atrevida y novedosa de la Comunidad Europea, con escasos precedentes. Merece la pena examinar, desde un punto de vista geográfico, la creación de una red pionera de microrreservas por parte del Servicio de Protección de Especies de la Generalitat Valenciana, con el fin de evaluar las implicaciones territoriales de su implantación.

Palabras clave: Planificación del medio físico, políticas de prevención y espacios naturales, protección de especies vegetales a escala de detalle.

\section{ABSTRACT}

Physical environment planning has got as priority objectives the application of a preventive policy capable to avoid the deteriorated environment. The preservation of environmental quality spaces supposes the cration of legal protection figures to guarantee its functional management. The political posture has changed during the history with economical criteria and influenced by cultural aspects and scientific evolution in the environmental knowledge. The apparition of the «microreserves of flora» in «Comunidad Valenciana» (mediterranean east coast of Spain) responds to a new stategy in the prtection matter based on scientific and ecological criteria as well as a daring and novel measure of European Community with scarcely precedents. Worthwhile to examine from a geographic point of view the creation of a pioneering net of microreserves by Species Protection Service of «Generalitat Valenciana» (Autonomous Government, ValenciaSpain) in order to evaluate the territorial implications.

Key words: Physical environment planning, prevention politics and natural spaces, flora protection a detail geographic scale. 


\section{Protección de espacios reducidos y conservación de especies}

La búsqueda de eficacia en nuestro sistema económico, desde un planteamiento racional, incluye la estimación de los costes sociales y ambientales de las actuaciones sobre el territorio. Los criterios físico ecológicos son los que deben determinar en primer lugar la organización del espacio y de las actividades humanas. La ordenación del territorio ha pasado de procurar el máximo beneficio a buscar el menor impacto sobre el medio ambiente, desde una posición de equilibrio de intereses. Ante esta situación, las actuaciones sobre el entorno deben ir precedidas de medidas preventivas o seguidas de acciones curativas. Toda vez que la mayor parte de los países prefieren incluir aquéllas en el planeamiento, con el fin de evitar los problemas económicos asociados a la regeneración, así como por la irreversibilidad de determinadas acciones humanas. El objetivo ideal sería llegar a una óptima utilización de los recursos sin menoscabo de la calidad de vida, integrando las políticas territoriales y ambientales.

La integración del estudio del medio físico y de sus recursos en la ordenación del territorio, desde hace unas décadas, junto a otros criterios sociales, culturales y económicos, ha dado lugar a lo que podríamos llamar planificación ambiental o planificación física. La postura preventiva aplicada en la planificación ambiental se sirve principalmente de una serie de instrumentos, entre los que destacaremos, por el tema que nos ocupa, la Política de Espacios Naturales Protegidos. Sólo teniendo en cuenta estos aspectos podremos comprender la implicación territorial de las microrreservas de flora y la importancia concedida por la Comunidad Europea a este tema, aunque sería necesario hacer un breve repaso previo de la historia reciente de los espacios protegidos en nuestro país.

La legislación y las actuaciones públicas sobre la conservación y protección de espacios naturales en España se remontan a principios del siglo actual. Desde entonces y hasta la actualidad se observa un cambio paulatino en los criterios, pasándose del ensalzamiento de los valores estéticos a la importancia científica y ecológica que pueden representar para la sociedad. Dentro del primer grupo se incluye la primera ley sobre estos aspectos en el país, la Ley de Parques Nacionales de 1916 cuyo fin era conseguir espacios dedicados a «la higienización y solaz de la raza, en que puedan tonificarse física y moralmente los cansados y consumidos por la ímproba labor y por respirar de continuo el aire viciado de las poblaciones». Esta quedaría completada con el Real-Decreto Ley de 1926 para proteger lugares pintorescos por parte de la Administración de Bellas Artes.

Hasta 1975 no se promulga una ley concreta sobre Espacios Naturales Protegidos teniendo las anteriores un carácter sectorial (Bases del Patrimonio Forestal del Estado de 1935, Ley de Montes de 1957, Ley de Caza de 1970, Ley del Suelo de 1976, Ley de Aguas de 1985, Ley de Costas de 1988). Dentro de las principales razones que justifican la conservación de estos espacios aparece una nueva concepción junto a la ya conocida lucrativa y paisajística que será el carácter científico, así como, por primera vez, la protección de especies en peligro de extinción: la ley de Conservación de los Espacios Naturales y de la Flora y Fauna Silvestres de 1989 que sustituyó a la anterior del '75 ya que no se ajustaba totalmente a las nuevas competencias del Estado y de las Autonomías y para ampliar la protección a las especies de flora y fauna. Pese a esta innovación, se sigue presentado la misma deficiencia, aunque en un grado menor, y es la excesiva burocracia del sistema que ralentiza el proceso de declaración de un espacio protegido. La Ley Valenciana reguladora de Parajes Naturales de la Comunidad Valenciana de 1988 trató de eliminar este obstáculo agilizando los trámites; aún así, todavía lentos.

La legislación existente hasta el momento oscila entre la protección de un espacio máximo y la especie en sí misma, ya sea vegetal o animal, obviando una escala intermedia 
como es la comunidad. Concretamente en el territorio valenciano la especie vegetal como tal queda protegida en la Orden del 20 de diciembre de 1985 de la Consellería de Agricultura y Pesca de Especies Endémicas o Amenazadas según el Real-Decreto 3091/1982 15 de octubre que prevé que las Comunidades Autónomas puedan publicar listas complementarias de plantas protegidas dentro de sus respectivos territorios en peligro de extinción por recolección incontrolada, uso comercial o ámbito reducido de distribución biogeográfica.

Los dos aspectos antes citados: procedimiento legal y escala de protección, hasta ahora no afrontados del todo, son junto al interés científico los pilares sobre los que se asienta la nueva figura de protección de especies silvestres o «microrreserva vegetal» creada por Decreto 218 del 17 de octubre de 1994 por el Gobierno Valenciano y definida como parcela inferior a las 20 ha en terreno público o privado, cuyo fin es la conservación para garantizar un estudio y desarrollo de actividades técnico-científicas. La conservación deja de ser un fin como hasta ahora, pasando a ser un medio.

El proceso legislativo se agiliza porque los límites de la microrreserva y el plan de gestión científico-técnico se establecen y aprueban al tiempo que su declaración. El inicio del expediente corresponde a la Dirección General de Conservación del Medio Natural o a petición de entidades o particulares, resolviéndose en tres meses.

Por lo tanto, surge como una nueva figura compatible y complementaria dentro de los Espacios Naturales Protegidos, pero con interesantes peculiaridades en sus fines: no sólo la conservación, sino también crear pequeños laboratorios naturales, bancos de germoplasma y fomentar la educación ambiental. En un futuro se pretende ampliar a la microfauna, geología y edafología. En la actualidad, la Dirección General está proponiendo a la Consellería de Medio Ambiente la creación de un Área exclusiva para la Conservación de la Flora dentro del Servicio de Protección de Especies.

\section{La creación de una red de microrreservas de flora en la Comunidad Valenciana}

El origen de este Decreto 218 se encuentra a finales de 1991 con la aceptación de la fase de prospección de terrenos para crear una red de microrreservas dentro del programa MAB de UNESCO. Más tarde, en 1993, se suscribe un contrato entre la CEE y la Generalitat Valenciana de cofinanciación con ayudas del fondo $\operatorname{LIFE}^{1}$ (900.000 ecus equivalentes a unos 150 millones de pesetas), vigente hasta 1997 para la creación de dicha red compuesta por 100 microrreservas.

Con esta figura legal de protección se da cabida a especies endémicas desde la escala provincial a la peninsular con una distribución muy localizada ya que sus hábitats suelen encontrarse poco evolucionados o degradados por la actividad humana debido a su escasa calidad ambiental (roquedos, canchales, saladares, dunas, sectores de pastoreo o de abandono del cultivo agrícola). Esto no significa que en un porcentaje pequeño no existan endemismos en espacios con mayor desarrollo vegetal, pero como demuestra la tabla I una gran parte corresponden al primer grupo, de ahí la necesidad de su conservación.

Se ha considerado un período de actuación menor a una década, hasta el año 2000, para consolidar una red de más de cien microrreservas, mediante tres fases, consecutivas pero superpuestas cronológicamente. La primera etapa, en plena conclusión, ha permitido la

1 LIFE es el instrumento financiero de apoyo a la política comunitaria en materia de medio ambiente. Fue adoptado en 1992 para cubrir un período de funcionamiento que concluyó en 1995. Un nuevo Reglamento, desde 1996, ha de servir para su vigencia hasta el año 2000. De esta manera, LIFE-Naturaleza debe contribuir a la aplicación de las Directivas Comunitarias «Aves» (79/409/CEE) y «Hábitats» (92/43/CEE), en especial, al establecimiento de una red europea de áreas protegidas: «NATURA 2000», cuyo objetivo es la conservación de los espacios que albergan las especies de flora y fauna más valiosas de la Unión Europea. 
Hábitat

Roquedos verticales, cingles, cintos

Ambientes pedregosos no verticales

Pastizales, herbazales y baldíos

Pastizales muy secos y matorrales bajos

Matorrales bajos (tomillares, salviares)

Saladares, yesares, ambientes salinos

Bosques de quercíneas y clímax forestales
Porcentaje $(\%)$

18.70

17.39

16.52

6.52

29.57

8.26

3.04

Fuente: Mateo, G. y Crespo, M.B. (1990): Claves para la flora valenciana. De. Del Cenia al Segura. Valencia.

declaración, hasta la fecha, de sesenta microrreservas, la mayor parte en terrenos estatales, de la Generalitat Valenciana o en montes de utilidad pública, teniendo previsto incluir veinticinco más. En la actualidad, estando en vigor la segunda fase, denominada «etapa de consolidación en suelo público», se pretende incluir terrenos de propiedad municipal hasta 1998. La tercera etapa, cuyo comienzo está previsto coincidiendo con el término de la anterior, se caracteriza por un objetivo muy claro: el acceso a los terrenos privados. Aunque se han comenzado ya algunos trámites en este sentido, pese a las dificultades de tipo presupuestario para realizar compensaciones de renta o subvenciones, la posibilidad de formas de financiación ingeniosas (activa/pasiva) ${ }^{2}$ o la vía del convenio con entidades bancarias, incluso universidades (por tratarse de titulares con intereses científicos o altruistas), podrían ser una garantía de éxito.

En el artículo cuarto del Decreto 218, referido al marco de protección, se enumeran las actividades prohibidas dentro de las microrreservas, entre las usuales para estos casos - basuras, extracciones de agua, suelo o rocas; recolección; acampada y tránsito; introducción de especies que puedan originar enfermedades o plagas- destaca la no supresión o limitación de las actividades ganaderas, agrícolas, forestales o de caza existentes con anterioridad a la declaración de esta figura de protección. La explicación a este hecho, que en principio puede parecer contradictorio, es que estas especies raras, endémicas o amenazadas, en la mayoría de los casos, deben su existencia a la permanencia durante años de estas actividades. Son plantas heliófilas que habitan terrenos degradados y pastizales o matorrales aclarados en sectores donde la presión ganadera, agrícola y/o incendios repetitivos han creado un medio que sólo es hostil para especies de estadios superiores en la sucesión vegetal.

Los planes de gestión de las microrreservas son sencillos, para agilizar su puesta en marcha, constituyendo responsabilidad de la Consellería de Medio Ambiente de la Generalitat Valenciana, que puede delegar en órganos colegiados para asesoramiento y coordinación. Elaborados por un equipo de topógrafos y botánicos, se procede a delimitar el área con una señalización provisional, luego se realiza un estudio detallado de las especies para ampliar o reducir esos límites, si procede, antes de terminar de preparar un completo material cartográfico.

No existe homogeneidad en los requisitos planteados para la gestión de las microrreser-

2 Realizar una protección, bien pasiva, subvencionada sin más, bien activa, financiando una inversión del propietario que participa, por tanto, en la administración y gestión del paraje. 
vas, dependiendo de las necesidades específicas de cada una. Esta medida es consecuente con la especificidad de parajes y especies, que impide la aplicación de modelos únicos, cuestión muy común en el ejercicio de la planificación del medio físico. No obstante, para asegurar un cierto grado de efectividad se procede a la revisión de los mismos a los 5 ó 10 años de funcionamiento, a medida que también se producen cambios lógicos en el entorno.

La puesta en marcha de red de pequeños espacios protegidos para la flora y su continuación en el futuro dependen, en gran medida, de la prolongación del fondo LIFE cuya vigencia finaliza en 1997, año que corresponde a la segunda etapa o de consolidación en suelo público. En las líneas de conservación de la flora, dentro del Programa de Conservación del Medio Natural de la Generalitat Valenciana, se dedica un 40\% de la dotación económica del Programa LIFE para la red de microrreservas de flora. En total, se dispone de unos 150 millones, capital que se destina a cuatro apartados presupuestarios principales: adquisición de terrenos de interés prioritario; conservación «in situ» o gestión; conservación «ex situ» (bancos de semillas,...), y por último difusión técnico-divulgativa.

El tercero de estos apartados es el que más dotación económica requiere, de modo que para el año '98 si el proyecto LIFE no prorroga o mantiene su grado de atención monetaria, será necesario destinar del presupuesto del Gobierno Valenciano un mínimo de 30 a 40 millones anuales para mantener el funcionamiento y consolidación de la red proyectada.

Los terrenos adquiridos hasta el momento han sido en su mayoría públicos, evitando los privados donde se corre el riesgo de encontrar oposición de particulares aislados e incluso de colectivos ${ }^{3}$, arropados por el propio Decreto 218/1994, cuyo artículo $3^{\circ}$, en su punto segundo, reza: «Salvo por petición expresa de sus propietarios, no podrán declararse microrreservas los terrenos de titularidad privada». La solución que está siendo adoptada hasta el momento por la Consellería consiste en eludir los parajes de titularidad privada y declarar zonas próximas de parecidas características topoecológicas e iguales especies vegetales, pero en suelo público. De todos modos, el Servicio de Protección de Especies de la Consellería ha realizado propuestas para obtener subvenciones en este apartado e incentivar a organismos no gubernamentales, como ya se ha referido al comienzo de este artículo.

\section{Análisis territorial de la distribución de microrreservas de flora}

Por el momento, los informes que están siendo objeto de trámite para su futura aprobación como «microrreservas de flora» suman un total de cincuenta para la provincia de Alicante (vid. tabla II). Si observamos con mayor detalle su distribución geográfica al sur de la Comunidad Valenciana, (mapa adjunto), resulta evidente su ausencia en las comarcas de l'Alacantí y L'Alcoià, mientras que abundan en las dos Marinas. El intento de buscar una explicación exclusivamente biogeográfica a este hecho ha sido un tanto estéril, lo mismo nos ocurre con el litoral desde Cabo de las Huertas hasta el Peñón de Ifach, destacando en este tramo Sierra Helada, y en el interior con la Sierra de la Solana y el conjunto Carrasqueta-Plans. Pero si se prescinde de un prejuicio fitosociológico, pueden encontrarse razones para elucidar esta situación, como ya veremos más adelante.

En el ámbito detallado de las comunidades protegidas es evidente el fuerte vínculo de las especies al medio donde se ubican, siendo ésta una de las premisas que caracterizan a

3 Incluso las declaraciones en terreno público pueden generar conflicto de intereses, un ejemplo lo encontramos en la Sierra de Salinas, en término de Villena, donde los agricultores «pobres» del municipio han gozado del usufructo de tierras pertenecientes al Ayuntamiento, bajo el compromiso de mantener la explotación activa. En esta zona, el colectivo implicado se muestra muy interesado en conseguir la titularidad de los terrenos que cultiva, con desconfianza manifiesta ante cualquier actuación municipal que no vaya en este sentido, lo que podría afectar a la microrreserva del paraje de la «Cueva del Lagrimal». 


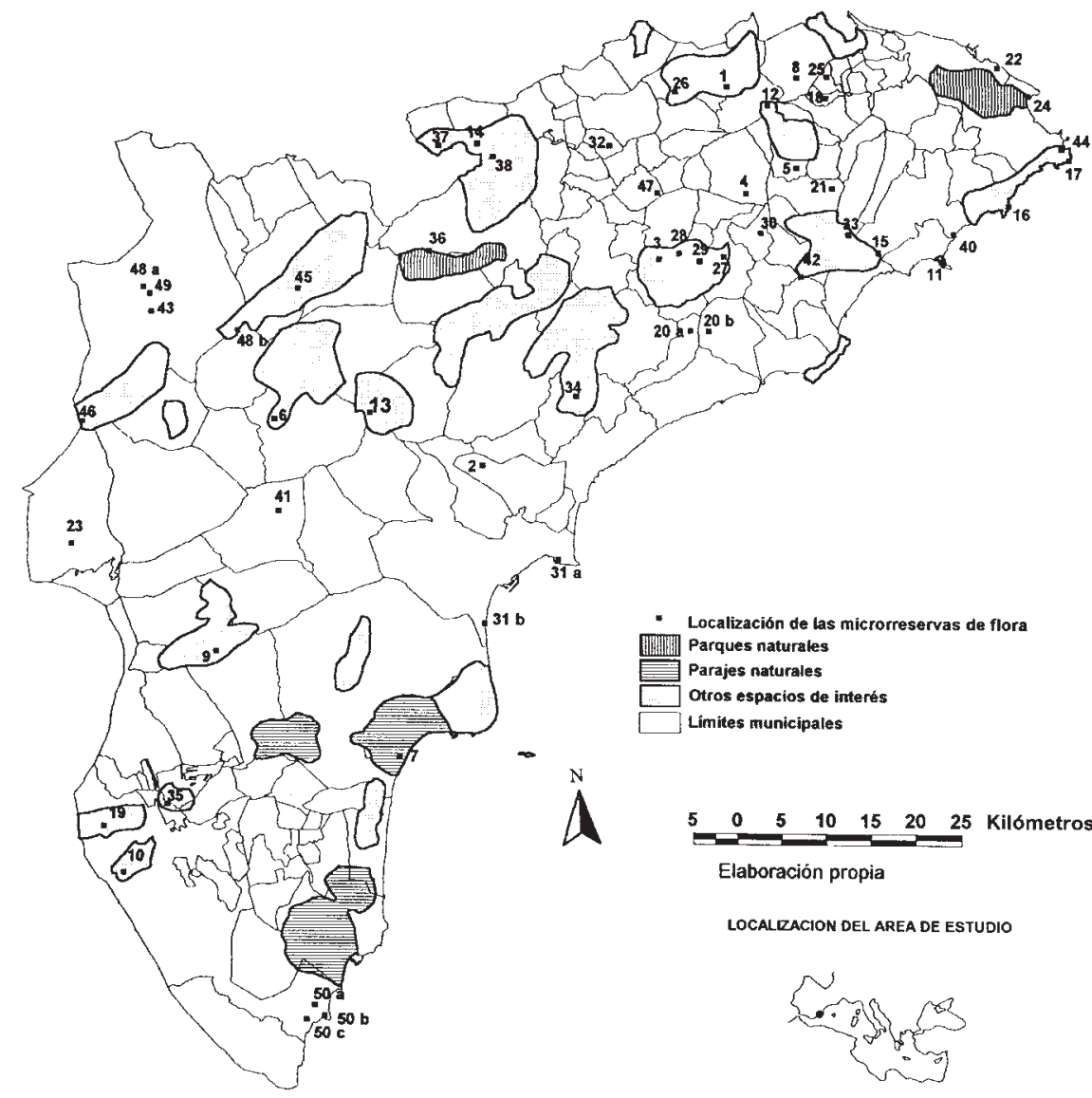

Tabla II

PARAJES PROPUESTOS PARA DECLARACIÓN COMO MICRORRESERVAS DE FLORA (PROVINCIA DE ALICANTE)

\begin{tabular}{|c|c|c|}
\hline $\begin{array}{l}\text { 1. Sa Aforadá: sector Benialí } \\
\text { 2. Cerro del Águila } \\
\text { 3. Sa Aitana: sector Confrides } \\
\text { 4. S Aixortá: sector Beniardá } \\
\text { 5. Peña del Altar } \\
\text { 6. El Arenal de Petrel } \\
\text { 7. Salinas de Bonmatí } \\
\text { 8. Sa Caval } \\
\text { 9. S Crevillente } \\
\text { 10. Monte Hurchillo } \\
\text { 11. Peñón de Ifach } \\
\text { 12. Barranco del Infierno } \\
\text { 13. Sa Maigmó } \\
\text { 14. Sa Mariola: Tejera de Agres } \\
\text { 15. El Mascarat } \\
\text { 16. Cabo de Moraira }\end{array}$ & $\begin{array}{l}\text { 17. Cabo de La Nao } \\
\text { 18. Valle de Orba } \\
\text { 19. Sa DE Orihuela: Rincón de Bonanza } \\
\text { 20. Orcheta-Finestrat } \\
\text { 21. Coll de Rates } \\
\text { 22. Las Rotas } \\
\text { 23. Cerro de la Sal } \\
\text { 24. Cabo de San Antonio } \\
\text { 25. S Segaria } \\
\text { 26. Sa Aforadà: sector Benisili } \\
\text { 27. S Aitana: sector Benimantell } \\
\text { 28. Sa Aitana: Fuente Partagás } \\
\text { 29. Sa Aitana: Pas de la Rabosa } \\
\text { 30. Sa Aixorta: Barranco las Águilas } \\
\text { 31. Alicante: Cabo Huertas y Cala Calabarda } \\
\text { 32. Sa Almudaina: sector Benialfaqui }\end{array}$ & $\begin{array}{l}\text { 33. Sa Bernia } \\
\text { 34. Sa Cabeçó d'Or } \\
\text { 35. Sa Callosa del Segura } \\
\text { 36. Sa Carrascal de Alcoy } \\
\text { 37. Sa Mariola: sector Alfafara } \\
\text { 38. Sa Mariola: Base Montcabrer } \\
\text { 39. Montgó: Cova de láigüa } \\
\text { 40. Moraira-Calpe } \\
\text { 41. La Nucia-Altea } \\
\text { 43. Salinas de Penalva } \\
\text { 44. Playa Portichol } \\
\text { 45. El Reconco de Biar } \\
\text { 46. Sa Salinas } \\
\text { 47. Sa Serrella } \\
\text { 48. Villena: Base Peña Rubia y Llanos de Lara } \\
\text { 49. Villena: Los Cabecicos } \\
\text { 50. La Zenia, Cabo Roig y Dehesa de Campoamor }\end{array}$ \\
\hline
\end{tabular}

Fuente: Generalitat Valenciana. Dirección General de Conservación del Medio Natural. Servicio de protección de Especies. 
las microrreservas, como auténticos reductos de flora. Pero en la revisión de estos microambientes hay uno que no se ha tenido en cuenta, el ripario, contando el sur de la Comunidad Valenciana con parajes de necesaria protección, por albergar especies del género Taray que están siendo eliminadas por la limpieza de acequias y el trazado de canalizaciones sobre cauces de ramblas y barrancos.

Esta revisión crítica, desde un punto de vista geográfico, tampoco pretende un mapa en el que la red de microrreservas se «densifique» hasta el punto de que cada municipio disponga de alguna de ellas. Lejos de esto, sólo se pretende hacer valer una tipología de parajes, quizá más compleja de proteger, pero que no por ello debe quedar condenada a la indefensión.

Esta visión territorial que supera la escala de detalle y nos introduce en unidades fisiográficas superiores, permitirá poner de manifiesto uno de los principales inconvenientes de esta figura de protección. Es muy fácil la declaración de zonas culminantes o divergentes dentro de una cuenca de drenaje, de roquedos inaccesibles, cantiles o estepas poco o nada «tensionadas» por la confluencia de actividades humanas, pero la cuestión es mucho más compleja en zonas húmedas, aguas abajo, en los llanos donde convergen los intereses humanos.

En estos últimos lugares, donde la baliza no basta para la protección del paraje, se hace difícil encontrar el método para evitar el peligro de impactos ambientales, inducidos e incluso directos, pues su complejidad de funcionamiento, su dependencia de factores exógenos y la concurrencia de actividades humanas más «agresivas», plantea un ejercicio de planeamiento del medio físico capaz de superar la escala de detalle. Además, la capacidad de acceder a la titularidad de espacios de alto valor socio-económico, partiendo de los presupuestos enunciados con anterioridad, resulta casi utópica, salvo que los convenios o acuerdos previstos con entidades financieras o universidades, como propietarias de los terrenos, sean capaces de «doblegar» su alta valoración en el mercado del suelo.

Por otro lado, existe un riesgo latente de generar una equivalencia locacional de microrreserva y municipio, es decir, suscitar la necesidad política para determinados ayuntamientos de tener que declarar este tipo de parajes protegidos. De hecho, si se observa la distribución de microrreservas por municipios del mapa adjunto, se pueden apreciar situaciones que merecen la pena ser resaltadas.

Así, hay municipios contiguos que han solicitado la declaración de sectores pertenecientes a la misma comunidad vegetal o con prácticamente los mismos endemismos: Sierra de Orcheta (Orcheta y Finestrat), Sierra Aitana (Confrides, Benimantell, Benifato), Sierra Aixortá (Beniardá, Benimantell, Guadalest) y Sierra Mariola (Agres, Alfafara, Cocentaina). También se da el caso de varias microrreservas en un mismo municipio: Jávea (Cabo de la Nao y Playa Portichol, sin problemas de conservación y ambos con el endemismo alicatino-balear Diplotaxis ibicensis); Villena (Base Peña Rubia, Llano de Lara, Salinas Penalva y los Cabecicos, los dos primeros pertenecen a la serie Sinrhamno lycioidisQuercetum cocciferae con especies similares y los otros dos con vegetación gipsícola sobre afloramientos del Keuper); Orihuela (La Zenia, Cabo Roig y Dehesa de Campoamor, especies litorales y halófilas).

La explicación a la distribución geográfica de la red de microrreservas, además de la simple existencia de especies susceptibles de protección, debe obedecer a criterios y medidas para el refuerzo de las poblaciones vegetales implicadas. De esta forma, la aparición en una misma sierra de parajes protegidos cercanos, casi de idénticas características, debe obedecer a una medida de salvaguarda frente a incendios forestales o incluso, a criterios de proximidad para favorecer la actuación de agentes polinizadores de cara a una óptima reproducción de especies. 


\begin{tabular}{|l|l|}
\hline Composición geomorfológica & Descripción topoecológica \\
\hline Acantilado & Acantilado \\
Barranco & Barranco \\
Barranco ancho & Cantil \\
Cantil & Cresta \\
Carretera abandonada & Cueva \\
Cingle & Erial \\
Colina & Ladera \\
Cresta & Saladar \\
Cueva & Terraza marítima \\
Cultivo de secano & \\
Derrubio rocoso & \\
Erial & \\
Glera & \\
Ladera & \\
Ladera con terrazas & \\
Ladera de colina & \\
Llano & \\
Llanura & \\
Matorral de ladera & \\
Matorral en llano & \\
Monte bajo & \\
Pie cantil & \\
Rellano & \\
Ribazo & \\
Roquedo & \\
Roquedo vertical & \\
Saladar & \\
Terraza & \\
Terraza desmonte & \\
Terraza desmonte & \\
Terraza marítima & \\
\hline
\end{tabular}

Fuente: Elaboración propia.

En este sentido, un mejor conocimiento del comportamiento biológico de estas especies y de su entorno, como resultado de su estudio científico, puede permitir en un futuro mejorar, si cabe, la localización y distribución de microrreservas de cara a la fecha de revisión de los planes de gestión. Esta faceta de conocer mejor el elemento biológico y territorial que se maneja, gracias a la utilización de los lugares protegidos como área de estudio, será doblemente útil en la fase de declaración de terrenos privados, en la que se puede obtener un altísimo grado de eficacia.

Otra situación, a escala supracomarcal, es la falta de coordinación legal entre comunidades autónomas, caso de la microrreserva de la Cueva del Lagrimal en la Sierra de Salinas (Villena), colindante con la Comunidad de Murcia. Esta unidad de relieve está considerada área natural protegida en la superficie que queda dentro de la autonomía vecina, mientras que en el sector valenciano no ocurre igual. La declaración de microrreserva no puede resolver, ni el vacío legal, ni la falta de definición del planeamiento para ejercer una protección efectiva de un espacio de calidad ambiental superior, amén de poner en evidencia una cierta incongruencia de tipo administrativo. 
El informe preliminar a la declaración de cada una de las microrreservas propuestas se estructura en tres partes. La primera, nos describe la zona de forma topográfica y la define en el sistema de coordenadas planas Transversal Universal de Mercator, estableciendo la extensión, altitud, orientación, inclinación, materiales geológicos y composición geomorfológica. En el segundo apartado, biogeográfico, enuncia la serie fitosociológica a la que pertenece para después listar las asociaciones propias de la zona. En último lugar, se adjuntan los inventarios realizados y se listan los endemismos encontrados (alicantinos, valencianos, alicantino-balear, murciano-almeriense, provincias limítrofes, iberolevantino y de amplia distribución).

Los endemismos alicantinos han sido inventariados en el Cerro del Águila (Vella lucentica), Arenal de Petrel (Helianthemum arenarium), Salinas de Bonmatí (Limonium santapolense, L. thiniense y L. furfuraceum), Monte Hurchillo (Teucrium buxifolium ssp rivasii), Orcheta-Finestrat (Teucrium lepicephalum), Las Rotas (Limonium rigualii), Cabo de las HuertasCala Calabarda (Limonium furfuraceum y L. lucentinum) y Playa Portichol (L. rigualii).

Acompaña al informe un apartado de material cartográfico a escalas 1:50.000 y 1:10.000, así como dibujos y croquis del sector en los que se localizan los inventarios efectuados. Es necesario matizar el término utilizado como «composición geomorfológica» en la primera parte de los informes preliminares, un tanto confuso y amplio, cuando podrían reducirse los 30 términos empleados para las 50 áreas, en 10 (vid. tabla III) y denominar como «descripción topoecológica».

\section{Los planes de gestión}

Se ha seleccionado el $10 \%$ de las microrreservas para un estudio detallado, analizando sus planes de gestión, ya aprobados. Estas áreas son los Parques Naturales del Peñón de Ifach, del Montgó y de la Font Roja (la microrreserva del Paraje Natural de las Salinas de Santa Pola todavía no tiene plan de gestión), la Cala de las Rotas, elegida por la presencia de una población importante de un endemismo alicantino (Limonium rigualii) y los Cabecicos de Villena como ejemplo de vegetación gipsícola:

- Peñón de Ifach: Han sido aprobadas dos microrreservas, una en su cara noreste y otra en la noroeste. Situadas dentro del Parque Natural declarado por Decreto 1/1987 de 19 de enero, tras ser comprado por la Generalitat Valenciana y reclasificado como Suelo No Urbanizable. La importancia florística de las microrreservas se explica por la presencia del endemismo valenciano Silene hifacensis que habiendo desaparecido durante años ha vuelto a rebrotar en las fisuras de las rocas del peñón. El peligro reside en la abundante afluencia de turistas, de ahí la necesidad de controlar y limitar este factor creándose senderos obligatorios.

- Cabo de San Antonio. Dentro del Parque Natural del Montgó, Decreto 25/1987 de 16 de marzo, el sector declarado como microrreserva pertenece al municipio de Jávea que lo clasifica como Suelo Rústico Agrícola, permitiendo de este modo el uso turísticoresidencial con viviendas aisladas. La microrreserva no encierra ninguna urbanización lo que ha permitido un estado de conservación excelente con gran interés florístico, ecológico y corológico, sobre todo por la presencia de Carduncellus dianus, endemismo alicantinobalear único en la península.

- Obac del Menejador. Su importancia es totalmente florística puesto que se incluye en el Parque Natural de la Font Roja, cuyo aprovechamiento controlado y regulado desde hace más de un siglo, ha permitido que las series Fraxino orni-Quercetum faginae y Quercetum rotundifoliae estén perfectamente desarrolladas. La microrreserva abarca parte de estas series y de vegetación rupícola.

- Cala de las Rotas. En el litoral de Denia a los pies de la Sierra del Montgó, pero 
fuera del Parque Natural. Es necesaria su protección por la elevada profusión turística y por el peligro de reducción e incluso de extinción de la población de Limonium rigualii, endemismo alicantino que se cifra en el sector entre 100-200 individuos.

- Los Cabecicos: En un principio la propuesta era de dos microrreservas, la del Salero y Los Cabecicos, pero la primera fue descartada por situarse en terreno privado, ya que en la segunda están representadas las mismas comunidades. Si bien, en años anteriores la presión ha sido importante (extracciones de piedra, pastoreo, remoción de terreno para reforestación con Pinus halepensis), lo que explica el matorral tan ralo y degradado de la zona, en la actualidad, la atenuación de estas actividades favoreciendo una recuperación importante de la cubierta vegetal que es eminentemente gipsícola, típica de sectores con afloramientos triásicos.

En los cinco planes de gestión se impide la urbanización y se exige el control de la afluencia turística que será informada debidamente por la colocación de las señales pertinentes para ello (Cabo de San Antonio y Peñón de Ifach). En lo que se refiere al tema científico, se establece un seguimiento de las poblaciones de cinco a diez años realizado por un biólogo (en la Font Roja y los Cabecicos) y la recolección de semillas para su cultivo en el Banco de Germoplasma del Jardín Botánico de Valencia. El coste de la señalización es de 200.000 pts., del seguimiento de 100.000 pts. y el tratamiento de las semillas de 40.000 pts. para Cabecicos y Font Roja, y de 500.000 pts. para Cabo de San Antonio y Peñón de Ifach (en cada caso), cifras superiores porque se incluye la posibilidad de otorgar ayudas y becas de investigación

Del análisis de los planes de gestión y como fruto del trabajo de campo, desde un punto de vista territorial, las microrreservas de flora que están en proceso de declaración en la provincia de Alicante se pueden repartir en tres grandes grupos:

- Un primer conjunto estaría formado por las cuatro que se encuentran dentro de figuras de protección de escala superior: los Parques Naturales del Peñón de Ifach, del Montgó y de la Font Roja y el Paraje Natural de las Salinas de Santa Pola. En todas ellas, con excepción de la Font Roja, existe una presión turística y urbanizadora no prohibida en el momento de su declaración legal, a finales de los ' 80 , por lo que se justifica la propuesta de protección de un área más pequeña dentro de la anterior. en donde existen especies endémicas amenazadas. Es evidente que el caso de la Font Roja no es éste, más bien, su declaración responde a un punto de vista científico, pues se trata del reducto de vegetación climácica mejor conservado en todo el sur de la Comunidad Valenciana.

- El segundo grupo, mucho más numeroso (como se pude apreciar en el mapa adjunto), sería el formado por aquéllas que se encuentran en el interior de espacios naturales de interés, la mayor parte de las veces protegidos por figuras menos estrictas. La declaración de microrreservas adquiere eficacia al restringir las actividades, configurar un elemento específico de protección y aportar un plan de gestión que mitiga la anterior situación de franco peligro para la preservación de las especies de interés. Además, la existencia de una valoración social, es decir, una cierta sensibilidad ambiental de tipo local hacia estos lugares más amplios, facilita todavía más la labor preventiva, es el caso de la Sierra del Maigmó, Sierra de Aitana, Cabeçó d'Or y otras tantas estribaciones montañosas señeras para las poblaciones alicantinas.

- Un último grupo, también numeroso, no sólo se ubica fuera de espacios protegidos o de parajes considerados de interés ambiental por el planeamiento local, incluso ocupan lugares conflictivos, como ocurre con Los Cabecicos de Villena, junto a antiguas canteras de yeso que han realizado el papel tradicional de «muladar», muelle de escombros y vertedero de basuras. En estos casos, la ausencia de una valoración por parte de la población y la tensión de usos justifican e imponen la declaración de protección, toda vez se vuelve más conflictiva y ve comprometida su eficacia. 


\section{El futuro de las microrreservas de flora}

A lo largo de este trabajo se ha justificado la creación de esta nueva figura de protección, mediante un breve repaso de la legislación, hasta llegar a la vigente; además de valorar su aportación a la política preventiva del planeamiento del medio físico. Posteriormente, se ha expuesto en qué consiste su funcionamiento y su desarrollo hasta la consolidación de una red de aproximadamente cien microrreservas en toda la Comunidad Valenciana, para proceder a un análisis en detalle de cinco de ellas, como requisito previo a la determinación de una agrupación tipológica provincial desde un punto de vista geográfico.

A modo de conclusión, merece la pena reseñar una serie de aspectos que induzcan, al menos, a la reflexión, con la modesta pretensión de aportar ideas que puedan ayudar a la cristalización funcional de un proyecto valiente e imaginativo, lejos, por tanto, de cualquier ánimo de crítica:

- La declaración de un área de protección menor a veinte hectáreas es muy útil para una política de protección con una gestión muy económica, pero puede ser poco eficaz en parajes con una caracterización topoecológica de «saladares», espacios húmedos, en lugares integrados en marcos fisiográficos superiores y dependientes de sistemas de mayor complejidad, escasamente representados en la red programada. Una solución sería declararlos en el interior de parques o parajes naturales acordes con esta caracterización fisiográfica, como en el caso de las Salinas de Santa Pola .

- El Decreto 218 centraliza la gestión de la red en la Consellería de Medio Ambiente para facilitar y agilizar trámites burocráticos, pero no desarrolla con detalle el papel de los Ayuntamientos en las microrreservas de su ámbito municipal. Sería necesario, incentivar actuaciones locales para evitar la relajación de su vigilancia y para generar una conciencia pública, en especial, en el tercero de los grupos anteriormente señalados, es decir, aquellas que se ubican fuera de otras áreas superiores de interés ambiental.

- La declaración de microrreservas en terrenos privados, pese a ser una fase del proyecto prevista con sumo cuidado, puede resultar un tema algo conflictivo, caso de no funcionar la política de convenios con organismos no gubernamentales o cuando la incentivación económica no sea capaz de atraer el interés de los propietarios del terreno. Siempre existe la posibilidad de buscar otra zona en terreno público que tenga las mismas comunidades vegetales, pero si ello no es factible, como puede ocurrir en el caso de emplazamientos peculiares, por ejemplo, una cueva, entonces habría que estimar la capacidad legal de realizar una expropiación, aspecto no recogido en el Decreto 218.

- La financiación puede quedar recortada de forma seria tras la finalización del proyecto LIFE en 1997, aspecto que debe ser contemplado con mucha cautela de cara a la consolidación de la red de microrreservas. Si revisamos la lista de propuestas para la provincia de Alicante, más de seis de cada diez corresponden a cantiles o lugares acantilados de difícil acceso y con especies rupícolas de gran similitud en la mayoría de los casos, cuando las plantas endémicas valencianas iberolevantinas sólo encuentran su hábitat óptimo en estos espacios en el $18,7 \%$ de los casos (vid. tabla I). No sería un desatino que conforme se fuesen completando las diferentes fases del programa, se realizase un trabajo de revisión de la distribución de la red, incluso contemplando la posibilidad de eliminación o la integración en áreas de protección superiores (parques naturales), siempre que se pudieran incluir los fines de la microrreserva en el plan de uso y gestión de la figura superior.

Sin embargo, pese a la idea que pueda sugerirse de la lectura anterior, la red de

4 El Plan de Gestión de la microrreserva de las Salinas de Santa Pola, en proceso de redacción, merece la pena ser estudiado con detalle, por ser prototípico para casos futuros en parajes tan complejos. 
microrreservas de flora en la Comunidad Valenciana, por el momento, no sólo surge como una figura de protección ingeniosa, meticulosa en su diseño y pionera dentro de la legislación existente en la Comunidad Europea sobre planificación del medio físico y política preventiva en la protección de espacios a escala de detalle. Además, se constituye como una vía eficaz de atraer importantes fondos de programas internacionales, valiosos por la faceta de preservación de especies y por hacer posible su estudio científico. En este sentido, la consolidación del programa para la protección de estos pequeños espacios puede constituir un rotundo éxito.

La solución a los «presuntos problemas» apuntados para la consolidación futura de la red de microrreservas depende, en parte, de seguir manteniendo la atención y el interés de nuestros socios europeos. Sin duda, el mejor conocimiento de estos lugares, fruto de los trabajos de investigación previstos, facilitará estudios de redistribución de la red, incluso la posibilidad de integrar objetivos y funciones en figuras de planeamiento superiores. La rentabilidad económica de estas medidas podría asegurar definitivamente el programa sin contar con el ingreso de los fondos internacionales.

El análisis territorial de la distribución de estos pequeños lugares protegidos podría servirse de la realización de un sistema de información geográfica, con la generación de una base de datos para la información específica del estado de cada microrreserva y de los resultados obtenidos de los estudios llevados a cabo en las mismas. La referenciación geográfica de estos datos permitiría realizar el cruce de variables temáticas y análisis topológicos, para estudios de riesgos frente a la agresión de incendios forestales, el grado de perniciosidad de determinadas actividades humanas, su situación frente a la evolución del planeamiento municipal o estudios de proximidad para evaluar la efectividad en el radio de acción de diferentes agentes polinizadores, entre otros.

Igualmente útil para la protección de estos lugares sería la incorporación de este sistema de información geográfica en el Sistema de Información Territorial de la Consellería de Obras Públicas, Urbanismo y Transportes, en el que ya se dispone de extensa información sobre vegetación actual y espacios de calidad ambiental.

Si se supera la falsa visión popular de esta zona del Mediterráneo deformada por el prejuicio del paisaje «verde», tan del gusto estético de la Europa central, poco a poco, gracias a este tipo de actuaciones públicas y a la profundización en el estudio de la biodiversidad y singularidad vegetal en ámbitos semiáridos, se podrá favorecer la percepción del valor ambiental del escenario «ocre», condigno a las características físicas de nuestro entorno.

\section{Bibliografía}

FUENTES BODELÓN, F. (1991): Ordenación del territorio y medio ambiente. Curso sobre: Planificación territorial y medio ambiente. Universidad Internacional Menéndez Pelayo. Valencia.

GONZÁLEZ BERNÁLDEZ, F. (1987): Los espacios protegidos y protegibles: los términos de una polémica. Coloquio Hispano-Francés de Espacios Protegidos. Casa Velázquez. Madrid.

GONZÁLEZ BERNÁLDEZ, F. (1991): «Integración de la ecología en los estudios urbanos y regionales». Curso sobre: Planificación territorial y medio ambiente. Universidad Internacional Menéndez Pelayo. Valencia.

LAGUNA LUMBRERAS, E. (1995): «Microrreservas de flora: un nuevo modelo de conservación en la Comunidad Valenciana». Quercus, dic., pp. 22-26, Madrid.

LAGUNA LUMBRERAS, E. Coord. (1995): Flora vascular rara, endémica o amenazada en la Comunidad Valenciana. Consellería de Medi Ambient. Generalitat Valenciana. Valencia.

LAGUNA LUMBRERAS, E. Coord. (1995): Conservación «in situ» de flora mediante microrreservas en la Comunidad Valenciana. Consellería de Medi Ambient. Generalitat Valenciana. Valencia.

PARRA SUPERVÍA, F. (1990): «La política de espacios naturales: una historia ambigua». Ciudad y Territorio, $\mathrm{n}^{\mathrm{o}} 83$, Madrid. 